TRANSACTIONS OF THE

AMERICAN MATHEMATICAL SOCIETY

Volume 362, Number 5, May 2010, Pages 2667-2684

S 0002-9947(09)05009-0

Article electronically published on November 17, 2009

\title{
PRIME NUMBERS IN LOGARITHMIC INTERVALS
}

\author{
DANILO BAZZANELLA, ALESSANDRO LANGUASCO, AND ALESSANDRO ZACCAGNINI
}

\begin{abstract}
Let $X$ be a large parameter. We will first give a new estimate for the integral moments of primes in short intervals of the type $(p, p+h]$, where $p \leq X$ is a prime number and $h=o(X)$. Then we will apply this to prove that for every $\lambda>1 / 2$ there exists a positive proportion of primes $p \leq X$ such that the interval $(p, p+\lambda \log X]$ contains at least a prime number. As a consequence we improve Cheer and Goldston's result on the size of real numbers $\lambda>$ 1 with the property that there is a positive proportion of integers $m \leq X$ such that the interval $(m, m+\lambda \log X]$ contains no primes. We also prove other results concerning the moments of the gaps between consecutive primes and about the positive proportion of integers $m \leq X$ such that the interval $(m, m+\lambda \log X]$ contains at least a prime number. The last applications of these techniques are two theorems (the first one unconditional and the second one in which we assume the validity of the Riemann Hypothesis and of a form of the Montgomery pair correlation conjecture) on the positive proportion of primes $p \leq X$ such that the interval $(p, p+\lambda \log X]$ contains no primes.
\end{abstract}

\section{INTRODUCTION}

Let $X$ be a large parameter, $\mathfrak{P}$ be the set of primes and $\lambda$ be a positive real number. This paper is devoted to studying the distribution of primes in short intervals: in particular we will give lower bounds for the proportion of positive integers $m \leq X$, or $p \in \mathfrak{P}$ and $p \leq X$, such that the intervals $(m, m+\lambda \log X]$ or $(p, p+\lambda \log X]$ contain or do not contain a prime number.

Many mathematicians have studied the distribution of primes in short intervals; here we just recall some fundamental papers on this topic. Several results are formulated using the quantity

$$
E=\liminf _{i \rightarrow+\infty} \frac{p_{i+1}-p_{i}}{\log p_{i}} .
$$

In 1926, Hardy and Littlewood [13, assuming the validity of the Generalized Riemann Hypothesis, gave the first non-trivial estimate $E \leq 2 / 3$. In 1940, Erdös [5] proved unconditionally that $E<1$, and in 1966, Bombieri and Davenport [1] improved this result to $E \leq 0.46650 \ldots$ In 1972-73, Huxley [15, 16], using a new set of weights, was able to reach $E \leq 0.44254 \ldots$. For all these results, a suitable modification of the argument can lead to a positive proportion result on the cardinality of the integers $m \leq X$ such that $(m, m+\lambda \log X]$ contains at least a prime number, for any fixed $\lambda$ larger than the given bound for $E$. In 1986, Maier used his matrix-method [18] to obtain $E \leq 0.2486 \ldots$, but this method gave no positive proportion results.

Received by the editors September 17, 2008.

2010 Mathematics Subject Classification. Primary 11N05; Secondary 11A41.

(C)2009 American Mathematical Society Reverts to public domain 28 years from publication 2667 
It was just in 2005 that Goldston, Pintz and Yıldırım [10] obtained that $E=0$, solving a long-standing conjecture. In fact they proved a quite stronger result:

there are infinitely many $i$ such that $p_{i+1}-p_{i} \ll \sqrt{\log p_{i}}\left(\log \log p_{i}\right)^{2}$.

Unfortunately, it seems that this wonderful new technique gives no positive proportion results.

In 1987, Cheer and Goldston 2, 3] used the integral moments of primes over integers and a refinement of Erdös's technique to prove results of this kind. We also recall that Goldston and Yıldırım [11, in a paper published in 2007 but that was developed before [10] appeared, were able to obtain a new proof of the inequality $E \leq 1 / 4$ with a method which also gives a positive proportion result.

Here we use a new result on integral moments of primes over primes (see Theorem 11) to prove that there exists a positive proportion of primes $p \leq X$ such that the interval $(p, p+\lambda \log X]$ contains at least a prime number for every $\lambda>1 / 2$; see Theorem 2, Even if the uniformity in $\lambda$ is weaker than the one proved in Theorem 1 of Goldston and Ylldırım [1] $(\lambda>1 / 4)$, here we obtain an evaluation of the implicit constant which will be useful in the consequences. The first of them is Theorem 3, in which we improve Cheer and Goldston's [2] result on the size of $\lambda>1$ such that there is a positive proportion of integers $m \leq X$ such that the interval $(m, m+\lambda \log X]$ contains no primes. The second consequence of Theorem 2 is a result concerning the moments of the gaps between consecutive primes (see Theorem 4).

Theorem 5 is about the positive proportion of primes $p \leq X$ such that the interval $(p, p+\lambda \log X]$, where $\lambda$ is "small", contains no primes and its Corollary 2 concerns the positive proportion of integers $m \leq X$ such that the interval $(m, m+\lambda \log X]$ contains at least a prime number. Our last result (Theorem 6) slightly refines a conditional theorem of Cheer and Goldston 2 on the positive proportion of primes $p \leq X$ such that the interval $(p, p+\lambda \log X]$, where $\lambda>0$, contains no primes.

To be more precise in describing our results we now need to give some notation and definitions. Let $1 \leq n \leq X / 2$ be an integer. We define the twin-prime counting functions as follows:

$$
Z(X ; 2 n)=\sum_{p \leq X} \sum_{\substack{p^{\prime} \leq X \\ p^{\prime}-p=2 n}} \log p \log p^{\prime} \quad \text { and } \quad Z_{1}(X ; 2 n)=\sum_{p \leq X} \sum_{\substack{p^{\prime} \leq X \\ p^{\prime}-p=2 n}} 1,
$$

where $p, p^{\prime} \in \mathfrak{P}$. Moreover we will write

$$
\mathfrak{S}(n)=2 c_{0} \prod_{\substack{p \mid n \\ p>2}} \frac{p-1}{p-2}, \quad \text { where } c_{0}=\prod_{p>2}\left(1-\frac{1}{(p-1)^{2}}\right)
$$

to denote the singular series for this problem and the twin-prime constant. Letting $1<K \leq X$ be a real number, we define

$\mathcal{A}(K)=\left\{p \leq X, p\right.$ prime, such that there exists a prime $p^{\prime}$ with $\left.0<p^{\prime}-p \leq K\right\}$,

$$
\mathcal{A}_{1}(K)=\{p \leq X, p \text { prime }\} \backslash \mathcal{A}(K),
$$

$\mathcal{B}(K)=\{m \in[1, X] \cap \mathbb{Z}$ such that there exists a prime in $(m, m+K]\}$

and

$$
\mathcal{B}_{1}(K)=[1, X] \backslash \mathcal{B}(K) .
$$


Moreover, let

$$
\mathcal{P}_{k}(y)=\sum_{r=1}^{k}\left\{\begin{array}{l}
k \\
r
\end{array}\right\} 2^{r} r ! y^{r}
$$

be a polynomial in $y$ where $\left\{\begin{array}{l}k \\ r\end{array}\right\}$ denotes the Stirling number of second type defined as the number of ways to partition a set with $k$ elements into $r$ non-empty subsets (not counting the order of the subsets).

Recalling that $\pi(u)$ is the number of primes up to $u$ and that $\psi(u)=\sum_{n<u} \Lambda(n)$, where $\Lambda(n)$ is the von Mangoldt function, we are now ready to state the following result concerning integral moments of primes over primes in short intervals. In what follows we will also denote by $\epsilon$ a small positive constant, not necessarily the same at each occurrence, and by $\omega \geq 1$ a parameter that will be useful in the applications.

Theorem 1. Let $\epsilon>0, \omega>1$, and $h \in \mathbb{R}, f(X) \leq h \leq X^{1-\epsilon}$, where $f(X) \rightarrow+\infty$ arbitrarily slowly as $X \rightarrow+\infty$. Further let $k \geq 2$ be an integer. Then

$$
\sum_{p \leq X}(\psi(p+h)-\psi(p))^{k} \leq\left(\mathcal{P}_{k+1}\left(\frac{\omega h}{\log X}\right)+\epsilon\right) \frac{X}{h(\omega-1)} \log ^{k} X,
$$

where $\mathcal{P}_{k}(y)$ is defined in (1.5).

The limitation to $\omega>1$ in Theorem 1 and in the following applications arises from Lemma 8 below. We are mainly interested in the case $h=\lambda \log X$, where $\lambda>0$ is a constant. Letting

$$
\mathcal{R}_{k, \omega}(\lambda)=\frac{\mathcal{P}_{k}(\omega \lambda)}{(\omega-1) \lambda}=\frac{1}{\omega-1} \sum_{r=1}^{k}\left\{\begin{array}{l}
k \\
r
\end{array}\right\} r ! 2^{r} \omega^{r} \lambda^{r-1},
$$

we have the

Corollary 1. Let $\epsilon>0, \omega>1$ and $k \geq 2$ be an integer. Further let $\lambda>0$ be a fixed constant. Then

$$
\sum_{p \leq X}(\psi(p+\lambda \log X)-\psi(p))^{k} \leq\left(\mathcal{R}_{k+1, \omega}(\lambda)+\epsilon\right) X \log ^{k-1} X
$$

and

$$
\sum_{p \leq X}(\pi(p+\lambda \log X)-\pi(p))^{k} \leq\left(\mathcal{R}_{k+1, \omega}(\lambda)+\epsilon\right) \frac{X}{\log X}
$$

Denoting by $|\mathcal{C}|$ the cardinality of a given set $\mathcal{C}$, we can now state our result on $|\mathcal{A}(K)|$ when $K$ is approximately $\log X$.

Theorem 2. Let $\epsilon>0, X$ be a large parameter and $\mathcal{A}(K)$ be defined as in (1.3). Further let $\lambda>1 / 2$ be a fixed constant. We have that

$$
|\mathcal{A}(\lambda \log X)| \geq\left(c_{1}(\lambda)-\epsilon\right) \frac{X}{\log X},
$$

where $c_{1}(\lambda)=\sup _{\ell \in \mathbb{Z} ; \ell \geq 2} \sup _{\omega>1} \Delta_{\ell, \omega}(\lambda)$ and

$$
\Delta_{\ell, \omega}(\lambda)=\frac{(\lambda / 2-1 / 4)^{\ell /(\ell-1)}}{\mathcal{R}_{\ell+1, \omega}(\lambda)^{1 /(\ell-1)}} .
$$


Theorem 2 means that there is a positive proportion of primes $p \leq X$ such that the interval $(p, p+\lambda \log X]$, with $\lambda>1 / 2$, contains at least a prime number. Our uniformity in $\lambda$ is weaker than the one in Theorem 1 of Goldston and Yildırım [11] $(\lambda>1 / 4)$, but there they gave no evaluation of the implicit constant. Since in the following applications we will need this, we have to use our weaker Theorem 2

Assuming a suitable form of the $k$-tuple conjecture, see, e.g., equation (3.2) below, it is clear that equation (4.3) below holds for every positive $\lambda$ and with the factor $\lambda / 2-1 / 4$ replaced by $\lambda / 2$. Hence in this case we can replace, in the statement of Theorem 2, the condition $\lambda>1 / 2$ with $\lambda>0$ and $\Delta_{\ell, \omega}(\lambda)$ with

$$
\widetilde{\Delta}_{\ell, \omega}(\lambda)=\frac{(\lambda / 2)^{\ell /(\ell-1)}}{\widetilde{\mathcal{R}}_{\ell+1, \omega}(\lambda)^{1 /(\ell-1)}},
$$

where

$$
\widetilde{\mathcal{R}}_{\ell, \omega}(\lambda)=\frac{1}{\omega-1} \sum_{r=1}^{\ell}\left\{\begin{array}{l}
\ell \\
r
\end{array}\right\} \omega^{r} \lambda^{r-1} .
$$

In fact a simpler form of the constant in Theorem 2 can be proved using $\omega=2$ and the Cauchy-Schwarz inequality instead of the Hölder inequality. But numerical computations proved, at least for $\lambda \in(1 / 2,2]$, that the largest constants $\Delta_{\ell, \omega}(\lambda)$ and $\widetilde{\Delta}_{\ell, \omega}(\lambda)$ are obtained with $\omega \approx 5503 / 5000$ and $\ell=11$ in the unconditional case, and for $\omega \approx 5939 / 5000$ and $\ell=10$ in the conditional case; moreover in the following application a different optimization is needed and the form in (1.7) is a more flexible one and leads to better final results. To write some numerical values, we have for $\lambda \in(1 / 2,2]$ that the largest $\Delta_{\ell, \omega}(\lambda)$ is about $0.01266456 \ldots$ while the largest $\widetilde{\Delta}_{\ell, \omega}(\lambda)$ is about $0.11604228 \ldots$.

As an application of Theorem 2 we have a result concerning the set $\mathcal{B}_{1}(K)$. We improve the estimates of Theorem 3 in Cheer and Goldston [2]. We also remark that, even if in [2] Cheer and Goldston used $p_{i} \in(X, 2 X]$ while we are working with $p_{i} \in(0, X]$, we still can compare the constants involved since the estimates have a good dependence on $X$.

Theorem 3. Let $\epsilon>0, X$ be a large parameter and $\mathcal{B}_{1}(K)$ be defined as in (1.4). Then there exists $\lambda>1$ such that

$$
\left|\mathcal{B}_{1}(\lambda \log X)\right| \geq\left(c_{2}(B, \lambda)-\epsilon\right) X
$$

where

$$
c_{2}(B, \lambda)=\sup _{\substack{\ell \in \mathbb{Z} ; \ell \geq 2 \\ \omega>1 \\ \nu \in(1 / 2,1-1 /(2 B))}} \frac{B\left(1-\lambda+\left(1-\Delta_{\ell, \omega}(\nu)\right)^{2} /(2 B)+(\lambda-\nu) \Delta_{\ell, \omega}(\nu)\right)^{2}}{2\left(1-\Delta_{\ell, \omega}(\nu)\right)^{2}}
$$

is a positive constant, $B$ is defined in Lemma 2 below and $\Delta_{\ell, \omega}(\nu)$ is defined in (1.7). Moreover we also have

$$
\sum_{p_{i} \leq X}\left(p_{i+1}-p_{i}\right)^{2} \geq\left(1+\frac{1}{12 B^{2}}+c_{3}(B)-\epsilon\right) X \log X
$$


where

$$
c_{3}(B)=\sup _{\substack{\ell \in \mathbb{Z} ; \ell \geq 2 \\ \omega>1 \\ \nu \in(1 / 2,1-1 /(2 B))}}\left[\frac{\left(1-\Delta_{\ell, \omega}(\nu)\right)^{3}}{3 B^{2}}-\frac{B}{3}\left(\frac{\Delta_{\ell, \omega}(\nu)(\nu-1)}{1-\Delta_{\ell, \omega}(\nu)}+\frac{2-\Delta_{\ell, \omega}(\nu)}{2 B}\right)^{3}\right]
$$

is a positive constant and $B$ and $\Delta_{\ell, \omega}(\nu)$ are as before.

The best $\lambda>1$ we are able to obtain in the previous statement is

$$
\begin{aligned}
\lambda= & \sup _{\substack{\ell \in \mathbb{Z} ; \ell \geq 2 \\
\omega>1 \\
\nu \in(1 / 2,1-1 /(2 B))}}\left(1-\Delta_{\ell, \omega}(\nu)\right)^{-1}\left(1-\nu \Delta_{\ell, \omega}(\nu)+\frac{\left(1-\Delta_{\ell, \omega}(\nu)\right)^{2}}{2 B}\right)-\epsilon \\
& \geq 1.145358 \ldots,
\end{aligned}
$$

where the numerical value is obtained using the estimate of Fouvry and Grupp [6] for $B=3.454, \nu \approx 0.666856, \ell=12$ and $\omega \approx 2491 / 2250$ in (1.7). Since it is not completely clear how to optimize the estimates in this theorem, it is possible that the numerical values written here and later can be further improved. We remark that Cheer and Goldston 2, in their Theorem 3, proved that $\lambda=1+1 /(2 B)$ is allowed in (1.10). Using the estimate for $B$ mentioned above, this leads to $\lambda=1.144759 \ldots$.

Moreover, we remark that the constant $c_{2}(B, \lambda)$ is larger than the one in eq. (3.3) of [2. For example, with $B=3.454$, for $\lambda=1+1 /(2 B)$ we get a gain of $\approx$ $6.1974568 \cdot 10^{-7}$ obtained for $\ell=12, \omega=2491 / 2250$ and $\nu=0.666856 \ldots$

In the proof of Theorem 3 we also show that (1.10) holds for

$$
\frac{1-\nu \Delta_{\ell, \omega}(\nu)}{1-\Delta_{\ell, \omega}(\nu)}-\frac{1-\Delta_{\ell, \omega}(\nu)}{2 B}<\lambda \leq \frac{1-\nu \Delta_{\ell, \omega}(\nu)}{1-\Delta_{\ell, \omega}(\nu)}+\frac{1-\Delta_{\ell, \omega}(\nu)}{2 B}
$$

thus extending, for $\Delta_{\ell, \omega}(\nu) \in(0,1)$ and $1 / 2<\nu<1-1 /(2 B)$, Cheer and Goldston's [2] result, which holds for $\lambda \in(1-1 /(2 B), 1+1 /(2 B)]$, to larger values of $\lambda$.

Finally, in (1.11) we get, for $\ell=12, \omega=2491 / 2250, \nu \approx 0.666323 \ldots$ and $B=$ 3.454 , the lower bound $1.00715710 \ldots$, which improves the value $1.00698512 \ldots$. in eq. (3.4) of Cheer and Goldston [2]; hence the constant $c_{3}(B)$ in (1.11) can be chosen as $c_{3}(B)=0.00017198 \ldots$. Now we assume a suitable form of the $k$-tuple conjecture, that is, equation (3.2) below, and, a fortiori, $B=1$ in Lemma 2. The remark after the statement of Theorem 2 implies that Theorem 3 and (1.12)-(1.13) still hold with the condition on $\nu$ replaced by $\nu \in(0,1 / 2)$ and $\Delta_{\ell, \omega}(\nu)$ replaced by $\widetilde{\Delta}_{\ell, \omega}(\nu)$ as defined in (1.8). In this case the numerical values for the key quantities are the following:

- $\lambda \geq 1.508146 \ldots$, for $\nu \approx 0.23435, \ell=10$ and $\omega \approx 39943 / 30000$, to be compared with $\lambda=1.5$ in [2];

- $c_{2}(1,3 / 2) \approx 3.3185202 \cdot 10^{-5}$ for $\nu \approx 0.23435, \ell=10$ and $\omega \approx 39943 / 30000$, to be compared with the value 0 in [2];

- (1.11) holds with the constant $1.09096653 \ldots$ obtained for $\nu \approx 0.22735$, $\ell=10$ and $\omega \approx 40027 / 30000$; this should be compared with $1.08333333 \ldots$ in [2. So we can choose $c_{3}(1)=0.0076331 \ldots$.

The next result is about an arbitrary positive power of $p_{i+1}-p_{i}$ whenever this distance is "small". We have the following. 
Theorem 4. Let $\epsilon>0, \alpha \geq 0, X$ be a large parameter and $\lambda>1 / 2$. Hence

$$
\sum_{\substack{p_{i} \leq X \\ p_{i+1}-p_{i} \leq \lambda \log X}}\left(p_{i+1}-p_{i}\right)^{\alpha} \geq\left(c_{4}(\lambda, \alpha)-\epsilon\right) X(\log X)^{\alpha-1},
$$

where for $\alpha>0$,

$$
c_{4}(\lambda, \alpha)=\sup _{\ell \in \mathbb{Z} ; \ell \geq 2} \sup _{\omega>1}\left(\frac{2 \alpha \Delta_{\ell, \omega}(\lambda)}{(\alpha+1) B}\right)^{\alpha} \frac{\Delta_{\ell, \omega}(\lambda)}{\alpha+1},
$$

$\Delta_{\ell, \omega}(\lambda)$ is defined in (1.7), $B$ is defined in Lemma 2 below and $c_{4}(\lambda, 0)=c_{1}(\lambda)$ is defined in Theorem 2 .

We remark that Theorem 4 collapses to Theorem 2 for $\alpha=0$ and that $c_{4}(\lambda, \alpha)$ is a decreasing function of $\alpha$. We also have a result concerning the set $\mathcal{A}_{1}(K)$ whenever $K$ is approximately $\log X$.

Theorem 5. Let $\epsilon>0, X$ be a sufficiently large parameter and $0<\lambda<2 / B-\epsilon$, where $B$ is defined in Lemma 2 below. We have that

$$
\left|\mathcal{A}_{1}(\lambda \log X)\right| \geq\left(1-\lambda \frac{(B+\epsilon)}{2}\right) \frac{X}{\log X} .
$$

Recalling the result in Fouvry and Grupp [6], see also the remark after Lemma 2, we can set $B=3.454$ and hence Theorem 5 holds for every $\lambda<0.579038 \ldots$. Assuming that the inequality in Lemma 2 holds with the best possible value $B=1$ we obtain that Theorem 5 holds for every $\lambda<2$. As a corollary we have

Corollary 2. Let $\epsilon>0, X$ be a sufficiently large parameter and $\lambda>0$. We have that

$$
|\mathcal{B}(\lambda \log X)| \gg_{\lambda, \epsilon} X .
$$

The implicit constant here is the same as in Theorem 5 for $\lambda<2 / B-\epsilon$ and it is $\epsilon$ otherwise.

Our last theorem is a conditional result on the cardinality of $\mathcal{A}_{1}(\lambda \log X)$ when $\lambda$ is not in the range described in Theorem [5.

Theorem 6. Let $X$ be a sufficiently large parameter. For $\lambda \geq 2 / B$, where $B$ is defined in Lemma 2 below, we assume that there exists a positive constant $c_{5}=c_{5}(\lambda)$ such that

$$
\sum_{\substack{p_{i} \leq X \\ p_{i+1}-p_{i}>\lambda \log X}}\left(p_{i+1}-p_{i}\right) \geq c_{5}(\lambda) X
$$

Assume further that there exists an absolute constant $c_{6}>0$ such that for every $\eta>\lambda$ we have

$$
\sum_{\substack{p_{i} \leq X \\ p_{i+1}-p_{i}>\eta \log X}}\left(p_{i+1}-p_{i}\right) \leq \frac{c_{6}}{\eta} X
$$

Then

$$
\left|\mathcal{A}_{1}(\lambda \log X)\right| \gg_{\lambda, \eta} \frac{X}{\log X}
$$


We remark that Heath-Brown proved that the hypothesis (1.15) holds under the assumption of the Riemann Hypothesis and of a suitable form of the Montgomery pair-correlation conjecture; see Corollary 1 of [14]. Theorem [6] should be compared with Theorem 5 of Cheer and Goldston [2] in which our hypothesis (1.14) is replaced by the stronger condition

$$
\sum_{\substack{p_{i} \leq X \\ p_{i+1}-p_{i}>\lambda \log X}}\left(p_{i+1}-p_{i}-\lambda \log X\right) \gg_{\lambda} X
$$

they used there. We finally remark that, using equation (5.5) below, we can also say that Theorem 5 of Cheer and Goldston [2] holds for $\lambda$ defined in (1.12).

\section{Main Lemmas}

We will use two famous results by Bombieri and Davenport.

Lemma 1 (Theorem 1 of Bombieri-Davenport [1]). Let $1 \leq T<(\log X)^{C}$ for some fixed positive $C$. Then, for any fixed positive $\epsilon$, we have

$$
\sum_{n=1}^{T} Z(X ; 2 n)>X \sum_{n=1}^{T} \mathfrak{S}(n)-\left(\frac{1}{4}+\epsilon\right) X \log X,
$$

where $Z(X ; 2 n)$ and $\mathfrak{S}(n)$ are defined in (1.1) $-(1.2)$.

Lemma 2 (Theorem 2 of Bombieri-Davenport [1]). There exists a positive constant $B$ such that, for any positive $\epsilon$ and for every positive integer $n$, we have

$$
Z(X ; 2 n)<(B+\epsilon) \mathfrak{S}(n) X,
$$

where $Z(X ; 2 n)$ and $\mathfrak{S}(n)$ are defined in (1.1) -(1.2), provided $X$ is sufficiently large.

Chen [4] proved that $B=3.9171$ can be used in Lemma 2. Recently Wu [22] slightly improved this by proving that $B=3.91045$ is admissible for every value of $n$. For $n \leq \log ^{A} X$, where $A>0$ is an arbitrary constant, the best result is $B=3.454$ by Fouvry and Grupp [6]. Moreover we remark that similar results hold for $Z_{1}(X ; 2 n)$, defined in (1.1), since $Z_{1}(X ; 2 n) \leq Z(X ; 2 n) \leq Z_{1}(X ; 2 n) \log ^{2} X$ and hence, using the inequalities

$$
\sum_{p \leq X / \log ^{4} X} \sum_{\substack{p^{\prime} \leq X \\ p^{\prime}-p=2 n}} \log p \log p^{\prime} \leq \pi\left(\frac{X}{\log ^{4} X}\right) \log ^{2} X=o\left(\frac{X}{\log ^{2} X}\right)
$$

and

$$
\sum_{X / \log ^{4} X<p \leq X} \sum_{\substack{p^{\prime} \leq X \\ p^{\prime}-p=2 n}} \log p \log p^{\prime}>(1+o(1)) \log ^{2} X \sum_{X / \log ^{4} X<p \leq X} \sum_{\substack{p^{\prime} \leq X \\ p^{\prime}-p=2 n}} 1,
$$

we also obtain

$$
\frac{Z(X ; 2 n)}{\log ^{2} X} \leq Z_{1}(X ; 2 n)<(1+o(1)) \frac{Z(X ; 2 n)}{\log ^{2} X}+o\left(\frac{X}{\log ^{2} X}\right) .
$$

Concerning the summation of the singular series of the twin-prime problem, we will use the following result. 
Lemma 3 (Friedlander-Goldston 7], eq. (1.13)). Let $X \geq 2$. We have

$$
\sum_{n \leq X} \mathfrak{S}(n)=X+\frac{1}{2} \log X+\mathcal{O}\left((\log X)^{2 / 3}\right),
$$

where $\mathfrak{S}(n)$ is defined in (1.2).

\section{Proof of Theorem 1 and Corollary 1}

We now define two different averages for primes in short intervals that we will need to prove Theorem 11. Let

$$
J_{k}(X, h)=\int_{0}^{X}(\psi(t+h)-\psi(t))^{k} \mathrm{~d} t \quad \text { and } \quad \widetilde{J}_{k}(X, h)=\sum_{m \leq X}(\psi(m+h)-\psi(m))^{k}
$$

be the Selberg integral and its discrete version. In the proof of Theorem 1 we will follow the line of Perelli and Salerno [19, 20] to connect the moments over primes with the corresponding ones over integers. To this end we now need several lemmas. We assume implicitly that $X$ is sufficiently large.

Lemma 4 (Gallagher [8, 9]). Let $\epsilon>0$ and $1 \leq h \leq X$. Further let $k \geq 2$ be an integer. Then

$$
\widetilde{J}_{k}(X, h) \leq\left(\mathcal{P}_{k}\left(\frac{h}{\log X}\right)+\epsilon\right) X \log ^{k} X
$$

where $\mathcal{P}_{k}(y)$ is defined in (1.5).

Proof. The proof follows immediately by inserting the following sieve estimate of Klimov [17] in Gallagher's argument (see also Theorem 5.7 of [12]):

$$
\sum_{m \leq X} \Lambda\left(m+h_{1}\right) \Lambda\left(m+h_{2}\right) \cdots \Lambda\left(m+h_{r}\right) \leq\left(2^{r} r !+\epsilon\right) X \mathfrak{S}\left(h_{1}, \ldots, h_{r}\right),
$$

where $h_{1}, \ldots, h_{r}$ are distinct integers such that $0 \leq h_{i} \leq h$ for every $i=1, \ldots, r$,

$$
\mathfrak{S}\left(h_{1}, \ldots, h_{r}\right)=\prod_{p}\left(1-\frac{1}{p}\right)^{-r}\left(1-\frac{\nu_{p}\left(h_{1}, \ldots, h_{r}\right)}{p}\right)
$$

and $\nu_{p}\left(h_{1}, \ldots, h_{r}\right)$ is the number of distinct residue classes modulo $p$ that the $h_{i}$, $i=1, \ldots, r$, occupy.

The case $k=4$ of Lemma 4 was recently proved by Goldston and Ylldırım; see eq. (7.32) of [11].

We also remark that, assuming the $k$-tuple conjecture in the form

$$
\sum_{m \leq X} \Lambda\left(m+h_{1}\right) \Lambda\left(m+h_{2}\right) \cdots \Lambda\left(m+h_{r}\right) \sim X \mathfrak{S}\left(h_{1}, \ldots, h_{r}\right) \quad \text { as } X \rightarrow+\infty,
$$

where $h_{1}, \ldots, h_{r}$ are distinct integers and $\mathfrak{S}\left(h_{1}, \ldots, h_{r}\right)$ is defined in (3.1), Gallagher [8] proved that Lemma 4 holds with the term $\mathcal{P}_{k}(h / \log X)$ replaced by $\widetilde{\mathcal{P}}_{k}(h / \log X)$, where

$$
\widetilde{\mathcal{P}}_{k}(y)=\sum_{r=1}^{k}\left\{\begin{array}{l}
k \\
r
\end{array}\right\} y^{r} .
$$

Now we see two lemmas on the connections between the Selberg integral and its discrete version. 
Lemma 5. Let $h$ be an integer, $1 \leq h \leq X$. Further let $k \geq 2$ be an integer. Then

$$
J_{k}(X, h)=\widetilde{J}_{k}(X, h)+\mathcal{O}\left(\frac{h^{k} \log ^{k} X}{\log ^{k}(2 h)}\right) .
$$

Proof. It is clear that $\widetilde{J}_{k}(X, h)=\widetilde{J}_{k}(\lfloor X\rfloor, h)=J_{k}(\lfloor X\rfloor, h)$. By the Brun-Titchmarsh inequality we have $J_{k}(X, h)=J_{k}(\lfloor X\rfloor, h)+\mathcal{O}\left(h^{k} \log ^{k} X(\log (2 h))^{-k}\right)$ and the lemma follows.

Lemma 6. Let $\epsilon>0$ and $h \in \mathbb{R} \backslash \mathbb{Z}$ with $1<h \leq X$. Further let $k \geq 2$ be an integer. Then

$$
\begin{aligned}
J_{k}(X, h) & =\widetilde{J}_{k}(X, h)+\mathcal{O}_{k}\left(X(\log X)^{k-1 / 2}\left(\mathcal{P}_{2 k-2}\left(\frac{2 h}{\log X}\right)+\epsilon\right)^{1 / 2}\right) \\
& +\mathcal{O}\left(\frac{h^{k} \log ^{k} X}{\log ^{k}(2 h)}\right)
\end{aligned}
$$

where $\mathcal{P}_{k}(y)$ is defined in (1.5).

Proof. By the Brun-Titchmarsh inequality and letting $h=\lfloor h\rfloor+\beta$ and $t=\lfloor t\rfloor+\tau$ we have

$$
\begin{aligned}
J_{k}(X, h) & =\int_{0}^{X}(\psi(\lfloor t\rfloor+\lfloor h\rfloor+\beta+\tau)-\psi(\lfloor t\rfloor+\tau))^{k} \mathrm{~d} t \\
& =\sum_{m \leq X} \int_{0}^{1}(\psi(m+\lfloor h\rfloor+\beta+\tau)-\psi(m))^{k} \mathrm{~d} \tau+\mathcal{O}\left(\frac{h^{k} \log ^{k} X}{\log ^{k}(2 h)}\right) \\
& =\sum_{m \leq X} \int_{0}^{1-\beta}(\psi(m+\lfloor h\rfloor)-\psi(m))^{k} \mathrm{~d} \tau \\
& +\sum_{m \leq X} \int_{1-\beta}^{1}(\psi(m+\lfloor h\rfloor+1)-\psi(m))^{k} \mathrm{~d} \tau+\mathcal{O}\left(\frac{h^{k} \log ^{k} X}{\log ^{k}(2 h)}\right) \\
& =(1-\beta) \widetilde{J}_{k}(X,\lfloor h\rfloor)+\beta \widetilde{J}_{k}(X,\lfloor h\rfloor+1)+\mathcal{O}\left(\frac{h^{k} \log ^{k} X}{\log ^{k}(2 h)}\right) .
\end{aligned}
$$

We have

$$
\begin{aligned}
\widetilde{J}_{k}(X,\lfloor h\rfloor+1) & -\widetilde{J}_{k}(X,\lfloor h\rfloor) \\
& =\sum_{m \leq X}\left((\psi(m+\lfloor h\rfloor+1)-\psi(m))^{k}-(\psi(m+\lfloor h\rfloor)-\psi(m))^{k}\right) .
\end{aligned}
$$

Let $a=\psi(m+\lfloor h\rfloor+1)-\psi(m)$ and $b=\psi(m+\lfloor h\rfloor)-\psi(m)$ : By the Mean Value Theorem we have $a^{k}-b^{k} \leq k a^{k-1}(a-b)$, since $b \leq a$. By the CauchySchwarz inequality, the Prime Number Theorem and the identity $\psi(m+\lfloor h\rfloor+1)-$ 
$\psi(m+\lfloor h\rfloor)=\Lambda(m+\lfloor h\rfloor+1)$, we get from Lemma 4 that

$$
\begin{gathered}
\widetilde{J}_{k}(X,\lfloor h\rfloor+1)-\widetilde{J}_{k}(X,\lfloor h\rfloor) \ll_{k}\left(J_{2 k-2}(X,\lfloor h\rfloor+1)\right)^{1 / 2}\left(\sum_{m \leq X} \Lambda(m+\lfloor h\rfloor+1)^{2}\right)^{1 / 2} \\
\ll_{k}(X \log X)^{1 / 2}\left[X \log ^{2 k-2} X\left(\mathcal{P}_{2 k-2}\left(\frac{\lfloor h\rfloor+1}{\log X}\right)+\epsilon\right)\right]^{1 / 2} \\
\ll X(\log X)^{k-1 / 2}\left(\mathcal{P}_{2 k-2}\left(\frac{2 h}{\log X}\right)+\epsilon\right)^{1 / 2} .
\end{gathered}
$$

Lemma 6 now follows because $\widetilde{J}_{k}(X, h)=\widetilde{J}_{k}(X,\lfloor h\rfloor)$.

Now let $u$ be a positive real number and

$$
\psi_{k}(X, u)=\sum_{\substack{m_{1}, \ldots, m_{k} \\ \min \left(m_{i}\right) \leq X \\ \max \left(m_{i}\right)-\min \left(m_{i}\right) \leq u}} \Lambda\left(m_{1}\right) \cdots \Lambda\left(m_{k}\right) .
$$

This function can be easily connected with the Selberg integral.

Lemma 7 (Perelli-Salerno [19, 20). Let $1 \leq h \leq X$, and $k \geq 2$ be an integer. Then

$$
J_{k}(X, h)=\int_{0}^{h} \psi_{k}(X, u) \mathrm{d} u+\mathcal{O}\left(\frac{h^{k+1} \log ^{k} X}{\log ^{k}(2 h)}\right) .
$$

Proof. Let $N=\max \left(m_{i}\right)$ and $n=\min \left(m_{i}\right)$ in (3.4). Expanding the $k$-th power in $J_{k}(X, h)$, we have

$$
\begin{aligned}
J_{k}(X, h) & =\sum_{\substack{m_{1}, \ldots, m_{k} \\
n \leq X \\
N-n \leq h}} \Lambda\left(m_{1}\right) \cdots \Lambda\left(m_{k}\right)(h-N+n) \\
& +\sum_{\substack{m_{1}, \ldots, m_{k} \\
X<n \leq X+h \\
N \leq X+h}} \Lambda\left(m_{1}\right) \cdots \Lambda\left(m_{k}\right)(X+h-N)=\Sigma_{1}+\Sigma_{2},
\end{aligned}
$$

say. By the partial summation formula we immediately get $\Sigma_{1}=\int_{0}^{h} \psi_{k}(x, u) \mathrm{d} u$ and, by the Brun-Titchmarsh inequality, we have $\Sigma_{2} \ll h^{k+1} \log ^{k} X(\log (2 h))^{-k}$. Lemma 7 follows.

The next lemma gives an upper bound for $\psi_{k}(X, h)$ in terms of the discrete Selberg integral.

Lemma 8. Let $\epsilon>0, \omega>1$ and $1 \leq h \leq X$. Further let $k \geq 2$ be an integer. Then

$$
\begin{aligned}
\psi_{k}(X, h) & \leq \frac{\widetilde{J}_{k}(X, \omega h)}{(\omega-1) h}+\mathcal{O}\left(\frac{\omega^{k+1} h^{k} \log ^{k} X}{(\omega-1) \log ^{k}(2 \omega h)}\right) \\
& +\mathcal{O}^{\prime}{ }_{k}\left(\frac{X \log ^{k-1 / 2} X}{(\omega-1) h}\left(\mathcal{P}_{2 k-2}\left(\frac{2 \omega h}{\log X}\right)+\epsilon\right)^{1 / 2}\right)
\end{aligned}
$$

where $\mathcal{O}^{\prime}$ means that this error term is present only if $h \notin \mathbb{Z}$ and $\mathcal{P}_{k}(y)$ is defined in (1.5). 
Proof. Since $\psi_{k}(X, u)$ is a positive and increasing function of $u$, it is easy to see that

$$
\psi_{k}(X, h) \leq \frac{1}{(\omega-1) h} \int_{h}^{\omega h} \psi_{k}(X, u) \mathrm{d} u \leq \frac{1}{(\omega-1) h} \int_{0}^{\omega h} \psi_{k}(X, u) \mathrm{d} u,
$$

where $\omega>1$ is a constant. By Lemmas 5, 6 and 7, we have

$$
\begin{aligned}
\int_{0}^{\omega h} \psi_{k}(X, u) \mathrm{d} u= & \widetilde{J}_{k}(X, \omega h)+\mathcal{O}\left(\frac{\omega^{k+1} h^{k+1} \log ^{k} X}{\log ^{k}(2 \omega h)}\right) \\
& +\mathcal{O}^{\prime}{ }_{k}\left(X \log ^{k-1 / 2} X\left(\mathcal{P}_{2 k-2}\left(\frac{2 \omega h}{\log X}\right)+\epsilon\right)^{1 / 2}\right)
\end{aligned}
$$

and hence Lemma 8 follows.

The following final lemma is a lower bound for $\psi_{k+1}(X, h)$ in terms of a weighted form of the discrete Selberg integral.

Lemma 9 (Perelli-Salerno [19, 20]). Let $1 \leq h \leq X$, and $k \geq 2$ be an integer. Then

$$
\sum_{m \leq X} \Lambda(m)(\psi(m+h)-\psi(m))^{k} \leq \psi_{k+1}(X, h) .
$$

Proof. First of all we remark that

$$
\sum_{m \leq X} \Lambda(m)(\psi(m+h)-\psi(m))^{k}=\sum_{m \leq X} \Lambda(m) \sum_{\substack{m_{1}, \ldots, m_{k} \\ m<m_{i} \leq m+h}} \Lambda\left(m_{1}\right) \cdots \Lambda\left(m_{k}\right) .
$$

Recalling now that $N=\max \left(m_{i}\right)$ and $n=\min \left(m_{i}\right)$ in (3.4), we trivially have

$$
\begin{aligned}
\psi_{k+1}(X, h) & \geq \sum_{n \leq X} \Lambda(n) \sum_{\substack{m_{2}, \ldots, m_{k+1} \\
m_{i} \neq n \\
0<N-n \leq h}} \Lambda\left(m_{2}\right) \cdots \Lambda\left(m_{k+1}\right) \\
& =\sum_{n \leq X} \Lambda(n) \sum_{\substack{m_{1}, \ldots, m_{k} \\
n<m_{i} \leq n+h}} \Lambda\left(m_{1}\right) \cdots \Lambda\left(m_{k}\right)
\end{aligned}
$$

and Lemma 9 follows immediately by combining (3.5) and (3.6).

Now we are ready to prove Theorem 1 and Corollary 1 .

By Lemmas 9, 8 and 4, the upper bound in the statement of Theorem 11 holds for the function $(\log X)^{-1} \sum_{m<X} \Lambda(m)(\psi(m+h)-\psi(m))^{k}$. It is easy to see that the contribution of $m=p^{\alpha}$ with $\alpha>1$ in the previous sum is negligible. Theorem 1 hence follows by the partial summation formula since $f(X) \leq h \leq X^{1-\epsilon}$, where $f(X) \rightarrow+\infty$ arbitrarily slowly as $X \rightarrow+\infty$. The first part of Corollary 1 can be obtained by inserting $h=\lambda \log X$ in Theorem 1 while the second part follows from the first one by using the partial summation formula.

\section{Proof of Theorem 2}

Let $X$ be a large parameter and $2 \leq K \leq X$. Our goal here is to elementarily prove a lower bound of the correct order of magnitude for the cardinality of the set 
$\mathcal{A}(K)$ defined in (1.3) when $K$ is approximately $\log X$. Let $\ell \geq 2$ be an integer. By the Hölder inequality we obtain

$$
\sum_{p \in \mathcal{A}(K)}(\pi(p+K)-\pi(p)) \leq\left(\sum_{p \in \mathcal{A}(K)}(\pi(p+K)-\pi(p))^{\ell}\right)^{\frac{1}{\ell}}|\mathcal{A}(K)|^{\frac{\ell-1}{\ell}}
$$

and hence

$$
|\mathcal{A}(K)| \geq\left[\sum_{p \in \mathcal{A}(K)}(\pi(p+K)-\pi(p))\right]^{\frac{\ell}{\ell-1}}\left(\sum_{p \in \mathcal{A}(K)}(\pi(p+K)-\pi(p))^{\ell}\right)^{-\frac{1}{\ell-1}}
$$

Now we proceed to estimate the term in the square brackets. It is easy to see that

$$
\sum_{p \in \mathcal{A}(K)}(\pi(p+K)-\pi(p))=\sum_{p \leq X}(\pi(p+K)-\pi(p))=\sum_{n=1}^{K / 2} Z_{1}(X ; 2 n),
$$

where $Z_{1}(X ; 2 n)$ is defined in (1.1), because $\pi(p+K)=\pi(p)$ by definition if $p \notin \mathcal{A}(K)$. From Lemmas 1 and 3 and (2.1), we get

$$
\sum_{n=1}^{K / 2} Z_{1}(X ; 2 n)>\frac{K X}{2 \log ^{2} X}+\frac{X \log (K / 2)}{2 \log ^{2} X}-\left(\frac{1}{4}+\epsilon\right) \frac{X}{\log X}+\mathcal{O}\left(X \frac{(\log (K / 2))^{2 / 3}}{\log ^{2} X}\right) .
$$

Hence, if $K=\lambda \log X$, with $\lambda>1 / 2$ and $X$ is sufficiently large, the previous remark implies

$$
\sum_{p \in \mathcal{A}(\lambda \log X)}(\pi(p+\lambda \log X)-\pi(p))>\left(\frac{\lambda}{2}-\frac{1}{4}-2 \epsilon\right) \frac{X}{\log X}
$$

To estimate the second term in (4.1), we use the second part of Corollary 1 with $k=\ell$. We immediately get

$$
\sum_{p \in \mathcal{A}(\lambda \log X)}(\pi(p+\lambda \log X)-\pi(p))^{\ell}=\sum_{p \leq X}(\pi(p+\lambda \log X)-\pi(p))^{\ell} \ll_{\ell, \lambda, \omega, \epsilon} \frac{X}{\log X},
$$

where the implicit constant is $\mathcal{R}_{\ell+1, \omega}(\lambda)+\epsilon$ and $\mathcal{R}_{\ell, \omega}(\lambda)$ is defined in (1.6). Now inserting (4.3)-(4.4) in (4.1) we obtain Theorem 2 with the implicit constant equal to $c(\ell, \lambda, \omega, \epsilon)=(\lambda / 2-1 / 4)^{\ell /(\ell-1)} \mathcal{R}{ }_{\ell+1, \omega}(\lambda)^{-1 /(\ell-1)}-\epsilon$ for every $\omega>1$ and every integer $\ell \geq 2$. Hence the best possible constant is $\sup _{\ell \in \mathbb{Z} ; \ell \geq 2} \sup _{\omega>1} c(\ell, \lambda, \omega, \epsilon)$.

Using the $k$-tuple conjecture, we can use (3.3) and, moreover, (4.3) holds with $\lambda / 2$ instead of $\lambda / 2-1 / 4$. Hence the implicit constant in this theorem is equal to $\widetilde{c}(\ell, \lambda, \omega, \epsilon)=(\lambda / 2)^{\ell /(\ell-1)} \widetilde{\mathcal{R}}_{\ell+1, \omega}(\lambda)^{-1 /(\ell-1)}-\epsilon$, where $\widetilde{\mathcal{R}}_{\ell, \omega}(\lambda)$ is defined in (1.9).

\section{Proof of Theorem 3}

Let $1 / 2<\mu<\lambda$ be real numbers. By the Prime Number Theorem we get $p_{\pi(X)+1}=X(1+o(1))$ and hence, following the line of Cheer and Goldston's proof 
of Theorem 1 in [2], we get that

$$
\begin{aligned}
& 1+o(1)=\frac{p_{\pi(X)+1}-2}{X}=\frac{1}{X} \sum_{p_{i} \leq X}\left(p_{i+1}-p_{i}\right) \\
&=\mu+\frac{1}{X}\left(-S_{X}(\mu)+\sum_{\substack{p_{i} \leq X \\
\mu \log X<p_{i+1}-p_{i} \leq \lambda \log X}}\left(p_{i+1}-p_{i}-\mu \log X\right)\right. \\
& \\
&\left.+\sum_{\substack{p_{i} \leq X \\
p_{i+1}-p_{i}>\lambda \log X}}\left(p_{i+1}-p_{i}-\mu \log X\right)\right)+o(1),
\end{aligned}
$$

where

$$
S_{X}(\mu)=\sum_{\substack{p_{i} \leq X \\ p_{i+1}-p_{i} \leq \mu \log X}}\left(\mu \log X-p_{i+1}+p_{i}\right) .
$$

Choose $\nu \in(1 / 2, \mu)$ : by Theorem 2 we have

$$
\begin{aligned}
S_{X}(\mu) \geq & \sum_{\substack{p_{i} \leq X \\
p_{i+1}-p_{i} \leq \nu \log X}}\left(\mu \log X-p_{i+1}+p_{i}\right) \\
> & (\mu-\nu) \log X \sum_{\substack{p_{i} \leq X \\
p_{i+1}-p_{i} \leq \nu \log X}} 1 \\
& >(\mu-\nu) \Delta_{\ell, \omega}(\nu) X,
\end{aligned}
$$

where $\Delta_{\ell, \omega}(\nu)$ is defined in (1.7). Inserting (5.2) in (5.1) and arguing again as on page 475 of Cheer and Goldston [2, we get

$$
1-\mu+(\mu-\nu) \Delta_{\ell, \omega}(\nu)-B \frac{(\lambda-\mu)^{2}}{2}-\epsilon \leq \frac{1}{X} \sum_{\substack{p_{i} \leq X \\ p_{i+1}-p_{i}>\lambda \log X}}\left(p_{i+1}-p_{i}-\mu \log X\right)
$$

and we gain the summand $(\mu-\nu) \Delta_{\ell, \omega}(\nu)$ comparing this equation with (3.8) in 2. Now we have to optimize the LHS of (5.3). If we consider $\lambda$ and $\nu$ fixed, then the maximum is attained for $\mu=\lambda-\left(1-\Delta_{\ell, \omega}(\nu)\right) / B$ and this, arguing as on page 476 of Cheer and Goldston 2, leads to

$$
\lambda<\left(1-\Delta_{\ell, \omega}(\nu)\right)^{-1}\left(1-\nu \Delta_{\ell, \omega}(\nu)+\frac{\left(1-\Delta_{\ell, \omega}(\nu)\right)^{2}}{2 B}\right)-\epsilon
$$

for every $\omega>1$ and every integer $\ell \geq 2$. Developing the right hand side in powers of $\Delta_{\ell, \omega}$, which is small, we see that we have

$$
\lambda<1+\frac{1}{2 B}+\Delta_{\ell, \omega}(\nu)\left(1-\frac{1}{2 B}-\nu\right)+\mathcal{O}\left(\Delta_{\ell, \omega}(\nu)^{2}\right) .
$$

For $1 / 2<\nu<1-1 /(2 B)$, our net gain over the result of Cheer and Goldston 2 is essentially in the third summand above, which we may maximize over $\omega, \ell$ and $\nu$.

Now we estimate $c_{2}(B, \lambda)$. First of all we recall that Gallagher, see the remark at the bottom of p. 87 of [14], proved that

$$
c_{2}(B, \lambda) \geq 1-\lambda,
$$

for any $\lambda \in(0,1)$. Now let $\lambda<\left(1-\Delta_{\ell, \omega}(\nu)\right)^{-1}\left(1-\nu \Delta_{\ell, \omega}(\nu)+\left(1-\Delta_{\ell, \omega}(\nu)\right)^{2} /(2 B)\right)$ $-\epsilon$ and $\tau \in \mathbb{R}, \tau \geq \lambda$. Starting again from (5.3), arguing as at the bottom of p. 476 
of [2], we get

$$
\begin{aligned}
& \quad \frac{1}{X} \sum_{\substack{p_{i} \leq X \\
p_{i+1}-p_{i}>\lambda \log X}}\left(p_{i+1}-p_{i}-\lambda \log X\right) \geq \\
& {\left[1-\mu+(\mu-\nu) \Delta_{\ell, \omega}(\nu)-B \frac{(\lambda-\mu)^{2}}{2}-\frac{B}{2}(\lambda-\mu)(\tau-\lambda)\right]\left(1+\frac{\lambda-\mu}{\tau-\lambda}\right)^{-1}-\epsilon .}
\end{aligned}
$$

Now, given $\lambda$ and $\nu$, we would like to maximize this term with respect to $\tau$ and $\mu$. Using the substitution $u_{1}=\lambda-\mu>0$ and $u_{2}=(\tau-\lambda) / u_{1} \geq 0$, the RHS of (5.5) becomes

$$
\left[1+u_{1}-\lambda-\frac{B}{2}\left(1+u_{2}\right) u_{1}^{2}+\left(\lambda-u_{1}-\nu\right) \Delta_{\ell, \omega}(\nu)\right] \frac{u_{2}}{u_{2}+1} .
$$

For $\Delta_{\ell, \omega}(\nu) \in(0,1)$ and $\lambda \in\left(\lambda_{1}, \lambda_{2}\right]$, where

$$
\lambda_{1}=\frac{1-\nu \Delta_{\ell, \omega}(\nu)}{1-\Delta_{\ell, \omega}(\nu)}-\frac{1-\Delta_{\ell, \omega}(\nu)}{2 B} \quad \text { and } \quad \lambda_{2}=\frac{1-\nu \Delta_{\ell, \omega}(\nu)}{1-\Delta_{\ell, \omega}(\nu)}+\frac{1-\Delta_{\ell, \omega}(\nu)}{2 B},
$$

equation (5.6) is maximized by

$$
u_{1}=\frac{1-\Delta_{\ell, \omega}(\nu)}{B\left(1+u_{2}\right)}
$$

and

$$
u_{2}=\frac{\left(1-\Delta_{\ell, \omega}(\nu)\right)^{2}-2 B\left(\lambda-1-(\lambda-\nu) \Delta_{\ell, \omega}(\nu)\right)}{\left(1-\Delta_{\ell, \omega}(\nu)\right)^{2}+2 B\left(\lambda-1-(\lambda-\nu) \Delta_{\ell, \omega}(\nu)\right)} .
$$

For these values of $u_{1}, u_{2}$, the maximal RHS of (5.5) is

$$
\frac{B}{2\left(1-\Delta_{\ell, \omega}(\nu)\right)^{2}}\left(1-\lambda+\frac{\left(1-\Delta_{\ell, \omega}(\nu)\right)^{2}}{2 B}+(\lambda-\nu) \Delta_{\ell, \omega}(\nu)\right)^{2},
$$

which is larger than (5.4) for every $\lambda \in\left(\lambda_{1}, \lambda_{2}\right]$, where $\lambda_{1}, \lambda_{2}$ are defined in (5.7).

Moreover, since $\Delta_{\ell, \omega}(\nu) \in(0,1)$ for $1 / 2<\nu<1-1 /(2 B)$, we have that equation (5.7) extends both the width of the $\lambda$-interval and the lower bound of eq. (3.3) of Cheer and Goldston 2 to larger values. Comparing again with eq. (3.3) of Cheer and Goldston [2], for $1 / 2<\nu<\lambda-1 / B$ our equation (5.8) gives a larger value for the final constant. This completes the proof of (1.10).

To prove (1.11) we follow again the line of the proof of Theorem 3 of Cheer and Goldston 2]; the only difference is paying attention to split the integration interval in eq. (3.10) there into the subintervals $[0,1-1 /(2 B)],\left[1-1 /(2 B), \lambda_{1}\right]$ and $\left[\lambda_{1}, \lambda_{2}\right]$, where $\lambda_{1}$ and $\lambda_{2}$ are defined in (5.7). We remark that $\lambda_{2} \geq 1+1 /(2 B)$ since $1 / 2<\nu<1-1 /(2 B)$. We have

$$
\begin{aligned}
\sum_{p_{i} \leq X}\left(p_{i+1}-p_{i}\right)^{2} & \geq 2(1-\epsilon) X \log X \int_{0}^{\lambda_{2}} c_{1}(B, \lambda) \mathrm{d} \lambda \\
& =(1-\epsilon) X \log X\left\{2 \int_{0}^{1-1 /(2 B)}+2 \int_{1-1 /(2 B)}^{\lambda_{1}}+2 \int_{\lambda_{1}}^{\lambda_{2}} c_{1}(B, \lambda) \mathrm{d} \lambda\right\} \\
& =(1-\epsilon) X \log X\left(I_{1}+I_{2}+I_{3}\right),
\end{aligned}
$$


say, where in the first integral we use Gallagher's estimate (5.4), in the second Cheer and Goldston's equation (3.3) from [2], and in the third our lower bound (5.8). A fairly tedious computation reveals that

$$
\begin{aligned}
& I_{1} \geq 1-\frac{1}{4 B^{2}}, \\
& I_{2} \geq \frac{1}{3 B^{2}}-\frac{B}{3}\left(\frac{\Delta_{\ell, \omega}(\nu)(\nu-1)}{1-\Delta_{\ell, \omega}(\nu)}+\frac{2-\Delta_{\ell, \omega}(\nu)}{2 B}\right)^{3}, \\
& I_{3} \geq \frac{\left(1-\Delta_{\ell, \omega}(\nu)\right)^{3}}{3 B^{2}} .
\end{aligned}
$$

Notice that $\Delta_{\ell, \omega}(\nu)=0$ yields exactly equation (3.4) in [2]. Developing again in powers of $\Delta_{\ell, \omega}$, we get

$$
I_{1}+I_{2}+I_{3} \geq 1+\frac{1}{12 B^{2}}+\frac{\Delta_{\ell, \omega}(\nu)}{B}\left(1-\frac{1}{2 B}-\nu\right)+\mathcal{O}\left(\Delta_{\ell, \omega}(\nu)^{2}\right) .
$$

Comparing this with equation (3.4) of 2, we see that our gain comes from the third term above, which is positive for $\nu<1-1 /(2 B)$. This completes the proof of Theorem 3 .

\section{Proof of Theorem 4}

Let $X$ be a large parameter and $0<\eta<\lambda$. First we remark that the case $\alpha=0$ corresponds to Theorem 2 So from now on we can assume $\alpha>0$. We have

$$
\begin{aligned}
\sum_{\substack{p_{i} \leq X \\
p_{i+1}-p_{i} \leq \lambda \log X}}\left(p_{i+1}-p_{i}\right)^{\alpha} & \geq \sum_{\substack{p_{i} \leq X \\
\eta \log X<p_{i+1}-p_{i} \leq \lambda \log X}}\left(p_{i+1}-p_{i}\right)^{\alpha} \\
> & (\eta \log X)^{\alpha} \sum_{\substack{p_{i} \leq X \\
\eta \log X<p_{i+1}-p_{i} \leq \lambda \log X}} 1 \\
& =(\eta \log X)^{\alpha}\left(\sum_{\substack{p_{i} \leq X \\
p_{i+1}-p_{i} \leq \lambda \log X}} 1-\sum_{\substack{p_{i} \leq X \\
p_{i+1}-p_{i} \leq \eta \log X}} 1\right) .
\end{aligned}
$$

For $\lambda>1 / 2$ we can apply Theorem 2, thus getting

$$
\begin{aligned}
\sum_{\substack{p_{i} \leq X \\
\eta \log X<p_{i+1}-p_{i} \leq \lambda \log X}}\left(p_{i+1}-p_{i}\right)^{\alpha} \\
\\
>(\eta \log X)^{\alpha}\left(\frac{X}{\log X}\left(\Delta_{\ell, \omega}(\lambda)-\epsilon\right)-\sum_{\substack{p_{i} \leq X \\
p_{i+1}-p_{i} \leq \eta \log X}} 1\right),
\end{aligned}
$$

where $\Delta_{\ell, \omega}(\lambda)$ is defined in (1.7). Now using equation (2.1) and Lemmas [2]3, we get

$$
\sum_{\substack{p_{i} \leq X \\ p_{i+1}-p_{i} \leq \eta \log X}} 1 \leq \sum_{n \leq(\eta / 2) \log X} Z_{1}(X, 2 n)<\frac{\eta}{2}(B+\epsilon) \frac{X}{\log X}
$$


and hence, by (6.1)-(6.2), we obtain

$$
\sum_{\substack{p_{i} \leq X \\ p_{i+1}-p_{i} \leq \lambda \log X}}\left(p_{i+1}-p_{i}\right)^{\alpha}>X \eta^{\alpha}(\log X)^{\alpha-1}\left(\Delta_{\ell, \omega}(\lambda)-\frac{\eta}{2}(B+\epsilon)\right) .
$$

Choosing the optimal value $\eta=2 \alpha \Delta_{\ell, \omega}(\lambda) /((\alpha+1) B)$ for $\alpha>0$, Theorem 4 follows.

\section{Proof of Theorem 5 and Corollary 2}

Let $X$ be a large parameter and $2 \leq K \leq X$. Arguing as in (4.2) we obtain

$$
|\mathcal{A}(K)|=\sum_{p \in \mathcal{A}(K)} 1 \leq \sum_{p \in \mathcal{A}(K)}(\pi(p+K)-\pi(p))=\sum_{n=1}^{K / 2} Z_{1}(X ; 2 n)
$$

and hence, by Lemmas 2 and 3 , we get

$$
|\mathcal{A}(K)| \leq(B+\epsilon) \frac{X}{\log ^{2} X} \sum_{n=1}^{K / 2} \mathfrak{S}(n)<(B+\epsilon) \frac{K X}{2 \log ^{2} X} .
$$

So we have that $\left|\mathcal{A}_{1}(K)\right|>\pi(X)-((B+\epsilon) / 2) X K(\log X)^{-2}$ and hence, letting $\lambda>0$ and $K=\lambda \log X$, we immediately get

$$
\left|\mathcal{A}_{1}(\lambda \log X)\right| \geq \pi(X)-(B+\epsilon) \lambda \frac{X}{2 \log X}>\left(1-(B+\epsilon) \frac{\lambda}{2}\right) \frac{X}{\log X}
$$

for every sufficiently large $X$. In the last inequality we have used $\pi(X)>X / \log X$ for every $X \geq 17$ proved in Corollary 1 of Rosser-Schoenfeld [21]. Choosing $\lambda<$ $2 / B-\epsilon$, Theorem 5 follows at once.

The proof of Corollary 2 runs as follows. The starting point is the trivial relation

$$
\begin{aligned}
|\mathcal{B}(K)|> & \sum_{\substack{p_{i} \leq X \\
p_{i}-p_{i-1}>K}} K+\sum_{\substack{p_{i} \leq X \\
p_{i}-p_{i-1} \leq K}}\left(p_{i}-p_{i-1}\right) \\
& =\sum_{\substack{p_{i} \leq X \\
p_{i+1}-p_{i}>K}} K+\sum_{\substack{p_{i} \leq X \\
p_{i+1}-p_{i} \leq K}}\left(p_{i+1}-p_{i}\right)+\mathcal{O}(K) .
\end{aligned}
$$

Letting $K=\lambda \log X$ and using Theorem [5, we immediately get

$$
|\mathcal{B}(\lambda \log X)|>\lambda \log X \sum_{\substack{p_{i} \leq X \\ p_{i+1}-p_{i}>\lambda \log X}} 1+\mathcal{O}_{\lambda}(\log X) \gg_{\lambda, \epsilon} X
$$

for every $0<\lambda<2 / B-\epsilon$. Corollary 2 now follows by remarking that $|\mathcal{B}(\lambda \log X)|$ is an increasing function of $\lambda$. 


\section{Proof of Theorem 6}

Let $\lambda \geq 2 / B$ and $\eta>\lambda$ be a real number. It is easy to see that

$$
\begin{aligned}
\sum_{\substack{p_{i} \leq X \\
p_{i+1}-p_{i}>\lambda \log X}} 1 \geq \frac{1}{\eta \log X} \sum_{\substack{p_{i} \leq X \\
\lambda \log X<p_{i}-p_{i} \leq \eta \log X}}\left(p_{i+1}-p_{i}\right) \\
=\frac{1}{\eta \log X}\left(\sum_{\substack{p_{i} \leq X \\
p_{i+1}-p_{i}>\lambda \log X}}\left(p_{i+1}-p_{i}\right)-\sum_{\substack{p_{i} \leq X \\
p_{i+1}-p_{i}>\eta \log X}}\left(p_{i+1}-p_{i}\right)\right) .
\end{aligned}
$$

Inserting the hypotheses (1.14) and (1.15) into (8.1) we immediately get

$$
\sum_{\substack{p_{i} \leq X \\ p_{i+1}-p_{i}>\lambda \log X}} 1>\frac{X}{\eta \log X}\left(c_{5}(\lambda)-\frac{c_{6}}{\eta}\right) .
$$

Theorem [6 now follows since, for any fixed $\lambda, \eta$ can be chosen greater than $\max \left(\lambda ; c_{6} / c_{5}(\lambda)\right)$.

\section{ACKNOWLEDGEMENTS}

We would like to thank Professors Heath-Brown and Maier for their insights and Professor Perelli for an interesting discussion about his joint papers with Salerno.

\section{REFERENCES}

1. E. Bombieri and H. Davenport. Small differences between prime numbers. Proc. R. Math. Soc., 293:1-18, 1966. MR0199165 (33:7314)

2. A.Y. Cheer and D.A. Goldston. Longer than average intervals containing no primes. Trans. Amer. Math. Soc., 304:469-486, 1987. MR911080(89d:11073)

3. A.Y. Cheer and D.A. Goldston. A moment method for primes in short intervals. C.R. Math. Rep. Acad. Sci. Canada, 11:101-106, 1987. MR880600(88e:11078)

4. J.R. Chen. On the Goldbach's problem and the sieve methods. Sci. Sinica, 21:701-739, 1978. MR517935 (80b:10069)

5. P. Erdős. The difference of consecutive primes. Duke Math. J., 6:438-441, 1940. MR0001759 $(1: 292 \mathrm{~h})$

6. É. Fouvry and F. Grupp. On the switching principle in sieve theory. J. Reine Angew. Math., 370:101-126, 1986. MR852513 (87j:11092)

7. J.B. Friedlander and D.A. Goldston. Some singular series averages and the distribution of Goldbach numbers in short intervals. Illinois J. Math., 39:158-180, 1995. MR1299655 (95i:11115)

8. P.X. Gallagher. On the distribution of primes in short intervals. Mathematika, 23:4-9, 1976 . MR0409385 (53:13140)

9. P.X. Gallagher. Corrigendum to: "On the distribution of primes in short intervals". Mathematika, 28:86, 1981. MR632799 (82j:10072)

10. D.A. Goldston, J. Pintz, and C.Y. Yıldırım. Primes in Tuples I. to appear in Ann. Math, 2005. http://arxiv.org/abs/math/0508185

11. D.A. Goldston and C.Y. Yıldırım. Higher correlations of divisor sums related to primes. III. Small gaps between primes. Proc. Lond. Math. Soc. (3), 95(3):653-686, 2007. MR 2368279 (2008i:11116)

12. H. Halberstam and H.-E. Richert. Sieve Methods. Academic Press, 1974. MR0424730 (54:12689)

13. G.H. Hardy and J.E. Littlewood. Some problems of Partitio Numerorum: VII. Unpublished, 1926.

14. D.R. Heath-Brown. Gaps between primes, and the pair correlation of zeros of the zeta-function. Acta Arith., 41:85-99, 1982. MR667711(83m:10078) 
15. M.N. Huxley. On the difference between consecutive primes. Invent. Math., 15:155-164, 1972. MR 0292774 (45:1856)

16. M.N. Huxley. Small differences between consecutive primes. Mathematika, 20:229-232, 1973. MR0352021 (50:4509)

17. N.I. Klimov. Combination of elementary and analytic methods in the theory of numbers. Uspehi Mat. Nauk (N.S.), 13:145-164, 1958. (Russian). MR0097372 (20:3841)

18. H. Maier. Small differences between prime numbers. Michigan Math. J., 35:323-344, 1988. MR978303 (90e:11126)

19. A. Perelli and S. Salerno. On an average of primes in short intervals. Acta Arith., 42:91-96, 1982. MR $679000(84 \mathrm{~b}: 10064)$

20. A. Perelli and S. Salerno. On $2 k$-dimensional density estimates. Studia Sci. Math. Hungar., 20:345-355, 1985. MR886039 (88f:11077)

21. J.B. Rosser and L. Schoenfeld. Approximate formulas for some functions of prime numbers. Illinois J. Math., 6:64-94, 1962. MR0137689(25:1139)

22. J. Wu. Chen's double sieve, Goldbach's conjecture and the twin prime problem. Acta Arith., 114(3):215-273, 2004. MR2071082 (2005e:11128)

Dipartimento di Matematica, Politecnico di Torino, Corso Duca degli Abruzzi 24, 10129 TORINO, ITALY

E-mail address: danilo.bazzanella@polito.it

Dipartimento di Matematica Pura e Applicata, Università di Padova, Via Trieste 63, 35121 PAdova, ItALY

E-mail address: languasco@math.unipd.it

Dipartimento di Matematica, Università di Parma, Parco Area delle Scienze, 53/A, Campus Universitario, 43100 Parma, Italy

E-mail address: alessandro.zaccagnini@unipr.it 